\title{
The effect of tidal volumes on the time to wean persons with high tetraplegia from ventilators
}

\author{
WP Peterson*,1, L Barbalata ${ }^{1}$, CA Brooks ${ }^{1}$, KA Gerhart $^{1}$, DC Mellick ${ }^{1}$ and GG Whiteneck ${ }^{1}$ \\ ${ }^{1}$ Craig Hospital, The Rocky Mountain Regional Spinal Injury Center, Englewood, Colorado, USA
}

\begin{abstract}
Study design: Retrospective review of patient medical records comparing the outcomes of two groups of individuals with high-level (C3-4) tetraplegia - those with high-volume and those with low-volume mechanical ventilation.

Objectives: To determine if higher volume mechanical ventilation contributes to faster weaning from the ventilator with fewer complications in individuals with ventilator dependent high-level tetraplegia.

Setting: A Model Spinal Injury Center, located in Colorado, USA, which treats patients referred from throughout the Rocky Mountain region as well as other states.

Methods: The medical records of 42 individuals with ventilator dependent C3-4 tetraplegia, admitted consecutively to the Rocky Mountain Regional Spinal Injury System between 1983 and 1993, were reviewed. All had required full-time mechanical ventilation upon admission, but had successfully weaned by discharge. They were divided into two groups that differed with respect to the ventilator tidal volumes used during their treatment and weaning: a large volume group ( $>20 \mathrm{mls} / \mathrm{kg}$ of body weight) and a smaller volume group $(<20 \mathrm{mls} / \mathrm{kg}$ of body weight).

Results: Though the two groups were equivalent in neurological level and completeness, muscular function, initial spontaneous vital capacity, the weaning method used (T-piece), and final spontaneous vital capacity, those in the large tidal volume group successfully weaned an average of 21 days faster than the lower tidal volume group (37.6 days $v s 58.7$ days, $P=0.02$ ). They also had significantly less atelectasis $(P=0.01)$ than the lower tidal volume group.

Conclusion: This research suggests that the use of higher ventilator tidal volumes may speed up the weaning process and lessen respiratory complications. Because of the potential for this to decrease the length and cost of the rehabilitation programs for persons with high-level tetraplegia, further large-scale research is needed to verify these single-center findings.
\end{abstract}

Keywords: respirator weaning; tetraplegia; ventilator; mechanical, weaning

\section{Introduction}

Only since the advent of modern resuscitation techniques and advanced trauma care have persons with high level spinal cord injuries begun to survive their initial injuries. Many of these individuals, however, require mechanical ventilation to combat severe respiratory complications or, because of paralysis of their respiratory musculature, to insure longer-term survival. Not only has survival among both groups increased dramatically over the past several decades, ${ }^{1}$ but most ventilator dependent tetraplegics-as many as $83 \%$ in one report-now ultimately wean from the mechanical ventilator and regain the ability to breathe on their own. ${ }^{2,3}$

*Correspondence: WP Peterson, Craig Hospital, The Rocky Mountain Regional Spinal Injury Center, 3425 South Clarkson Street, Englewood, Colorado 80110, USA

The opinions contained in this publication are those of the authors and do not necessarily reflect those of the US Department of Education.
However, such respiratory-compromised tetraplegia survivors frequently present an unparalleled weaning challenge. The length of time required for them to become ventilator-free can be protracted-as long as 14 months in one report, ${ }^{4}$ up to 8 years in another. ${ }^{5}$ During this process (regardless of its duration) rehabilitation and community placement options are limited, and costly equipment and considerably higher levels of nursing, respiratory, and other specialized care are needed.

Not surprisingly there is substantial motivationparticularly as our health care systems undergo close scrutiny and focuses on cost containment - to shorten weaning times as much as possible, and many different techniques have been utilized to facilitate this process. These include various methods of weaning, the addition of such auxiliaries as continuous positive airway pressure (CPAP), positive end expiratory pressure (PEEP), and pressure support, as well as the 
use of larger ventilator volumes. The latter, however, has remained a highly controversial therapeutic approach. Despite some reports of efficacy, ${ }^{6-8}$ large tidal volumes (those greater than $15 \mathrm{ml} / \mathrm{kg} /$ body weight) have been linked to such severe and sometimes irreversible complications as barotrauma, bronchopleural air leak, pulmonary parenchymal damage, pulmonary edema, altered hemodynamics, decreased vital capacity, decreased compliance, and altered perfusion ratios. ${ }^{9-14}$

However, these complications are documented primarily in non-spinal cord injured subjects with active lung disease or pathology. Moreover, other researchers have shown that, despite these potential complications, larger ventilator volumes may be advantageous in clearing atelectasis ${ }^{15-18}$-a frequent complication in high level spinal cord injury survivors and a deterrent to their successful weaning. Clearly, research specifically addressing the weaning process in spinal cord injured persons is lacking. It was this paucity of spinal cord injury-specific data, as well as clinical experience at our center which seemed to suggest a role for gradually increasing, high volume ventilation, that led to the undertaking of the study reported here. Its purpose was to determine whether larger tidal volume settings on ventilators do in fact contribute to faster weaning from the ventilator and fewer pulmonary complications in individuals with tetraplegia.

\section{Methods}

The subjects for this study consisted of 42 individuals with C3-4 tetraplegia consecutively admitted for initial rehabilitation at the Rocky Mountain Regional Spinal Injury System (RMRSIS) between 1983 and 1993. Individuals with complete tetraplegia at the $\mathrm{C} 3$ and $\mathrm{C} 4$ neurological levels were selected because they represent a level at which ventilator free breathing is difficult but theoretically possible. ${ }^{2,5}$ Individuals with these levels of injury were identified by the presence of functional bilateral trapezius muscle strength (manual muscle test grades of 'fair' or better ${ }^{19}$ ), and non-functional distal musculature (grade 'poor' or less ${ }^{19}$ ), including both biceps and deltoids.

All eligible participants required total, $24 \mathrm{~h}$ a day ventilator support upon admission to the RMRSIS, but had successfully weaned by the time they were discharged. By examining only those patients who successfully weaned, it was possible to focus on those factors which influenced the weaning process itself.

The research design consisted of a retrospective medical record review comparing two groups of individuals: those for whom higher ventilator tidal volumes were used were compared to those for whom lower tidal volumes were used. To establish the two groups, ventilator tidal volume settings at two weeks post admission were used, expressed as milliliters $(\mathrm{ml})$ per kilogram $(\mathrm{kg})$ of ideal body weight. Ideal rather than actual body weight was utilized because of the rapid weight loss and muscular atrophy, especially in the lower extremities, experienced by many spinal cord injury survivors.

Those individuals who, at 2 weeks post-admission, had ventilator inspiratory tidal volumes greater than $20 \mathrm{ml} / \mathrm{kg}$ - a level at which previous research had shown more rapid clearing of atelectasis ${ }^{10}$-comprised the higher tidal volume (HTV) group $(n=19$; mean $=25.3 \mathrm{ml} / \mathrm{kg}$; range $=20.3-32.2 \mathrm{ml} / \mathrm{kg}$ ). The low tidal volume (LTV) group consisted of 23 individuals with ventilator inspiratory tidal volumes less than $20 \mathrm{ml} / \mathrm{kg} \quad$ (mean $=15.5 \mathrm{ml} / \mathrm{kg} ; \quad$ range $=11.6-$ $19.4 \mathrm{ml} / \mathrm{kg}$ ). At this 2 week cut-point, the groups did not differ significantly with respect to how far along in the weaning process they were (Table 1).

All members of both groups had been ventilated with assist/control ventilation, using a decelerating ramp flow pattern. Rates were typically 10-12 breaths/min, but had been increased in a few cases to prevent the patient from triggering or overriding the ventilator, or to minimize patient complaints of discomfort. Throughout the process, dead space between the ventilator and the tracheostomy was adjusted as needed to maintain $\mathrm{pCO}_{2}$ levels between 30 and 35 , a range that typically eliminates the patients' stimulus to trigger the ventilator without allowing the $\mathrm{pCO}_{2}$ to be too low. Initially, a partial air leak around the tracheostomy cuff-created by deflating the cuff fully or partially as tolerated by the patient-was used to permit vocalization; as each individual's weaning progressed, cuffs were deflated for longer periods of time and 'trach talks' were used not only to further enhance vocalization, but to assist with coughing as well. Typically-and especially as long as atelectasis persisted-tidal volumes were increased by $100 \mathrm{ml} /$ day $(50 \mathrm{ml} /$ day in patients under $168 \mathrm{~cm}$ tall) and flow rates were increased by $101 / \mathrm{min}$ daily $(5 \mathrm{l} / \mathrm{min}$ in patients under $168 \mathrm{~cm}$ tall), unless either caused peak pressures to exceed $40 \mathrm{cmH}_{2} \mathrm{O}$. If the latter occurred, increases were not made. Finally, in keeping with protocols for ventilator management at the RMRSIS, positive end expiratory pressure (PEEP) was not used in either group. ${ }^{20}$

The frequencies of complications throughout the hospitalization were tracked by thorough review of the complete medical record in order to document occurrences of: new chest tubes, pneumonia, and adult respiratory distress syndrome (ARDS). The presence or absence of atelectasis was based on radiologists' readings of chest X-rays. It was coded as 'new onset' when it appeared in individuals who had had no atelectasis at the time of admission to the RMRSIS.

Though specific patient readiness to begin weaning was determined by the attending pulmonologist, a patient vital capacity of at least $150 \mathrm{ml}$ was generally a prerequisite. Other key RMRSIS criteria for beginning and continuing weaning typically included: clear or improving chest radiographs, stable arterial blood gases, stable vital signs (especially heart rate and 
Table 1 Comparison of group characteristics and outcomes

\begin{tabular}{|c|c|c|c|c|c|c|c|}
\hline & Low tidal $v$ & olume group & $(\mathrm{n}=23)$ & High tidal v & olume group & $(\mathrm{n}=19)$ & \\
\hline & & Range & $\begin{array}{l}\text { Standard } \\
\text { deviation }\end{array}$ & Mean & Range & $\begin{array}{l}\text { Standard } \\
\text { deviation }\end{array}$ & $\mathrm{P}=$ \\
\hline Age at admission to RMRSIS & 29 years & $15-59$ & 11.86 & 31 years & $16-60$ & 13.85 & 0.70 \\
\hline $\begin{array}{l}\text { Duration of injury, at admission } \\
\text { to RMRSIS }\end{array}$ & 56.0 days & $0-117$ & 31.88 & 48.6 days & $14-178$ & 6.30 & 0.50 \\
\hline Spontaneous vital capacity & & & & & & & \\
\hline At admission & 0.631 & $0.18-1.5$ & 0.34 & 0.701 & $0.15-2.4$ & 0.55 & 0.63 \\
\hline At end & 1.51 & $0.70-2.50$ & 0.50 & 1.51 & $0.65-2.30$ & 0.53 & 0.56 \\
\hline Ventilator tidal volume & & & & & & & \\
\hline At DC from referring hospital & 0.951 & $0.65-1.20$ & 0.19 & 0.921 & $0.60-1.90$ & 0.31 & 0.75 \\
\hline Day 1 at RMRSIS & 1.011 & $0.60-1.20$ & 0.16 & 1.081 & $0.60-2.00$ & 0.31 & 0.38 \\
\hline 2 weeks at admission to RMRSIS & 1.181 & $0.85-1.70$ & 0.19 & 1.731 & $1.10-2.2$ & 0.31 & $<0.001$ \\
\hline Peak ventilator flow rates & & & & & & & \\
\hline Day 1 at RMRSIS & $70.0 \mathrm{l} / \mathrm{min}$ & $60-90$ & 8.53 & $74.4 \mathrm{l} / \mathrm{min}$ & $50-120$ & 14.64 & 0.231 \\
\hline $\begin{array}{l}2 \text { weeks at admission to } \\
\text { RMRSIS }\end{array}$ & $76.30 \mathrm{l} / \mathrm{min}$ & $52-125$ & 18.41 & $91.11 \mathrm{l} / \mathrm{min}$ & $60-120$ & 19.61 & 0.02 \\
\hline Peak pressure & $28.83 \mathrm{cmH}_{2} \mathrm{O}$ & $18-35$ & 5.09 & $35.2 \mathrm{cmH}_{2} \mathrm{O}$ & $28-45$ & 5.11 & $<0.001$ \\
\hline Dynamic compliance & $42 \mathrm{ml} / \mathrm{cmH}_{2} \mathrm{O}$ & $29-60$ & 8.07 & $50 \mathrm{ml} / \mathrm{cmH}_{2} \mathrm{O}$ & $29-75$ & 11.7 & 0.013 \\
\hline Days until wean started & 8 days & $1-23$ & 6.99 & 4.82 days & $0-27$ & 7.63 & 0.19 \\
\hline Time from admission to wean & 58.7 days & $15-118$ & 33.03 & 37.6 days & $5-85$ & 21.38 & 0.02 \\
\hline$\%$ of participants with atelectasis & & & & & & & \\
\hline At admission to RMRSIS & $39 \%(n=9)$ & & & $84 \%(n=16)$ & & & 0.003 \\
\hline At start of wean & $48 \%(n=11)$ & & & $53 \%(n=10)$ & & & 0.80 \\
\hline $\begin{array}{l}2 \text { weeks at admission to } \\
\text { RMRSIS }\end{array}$ & $52 \%(n=12)$ & & & $16 \%(n=3)$ & & & 0.01 \\
\hline $\begin{array}{l}\text { Onset new atelectasis, in those } \\
\text { who were clear at admission }\end{array}$ & $\begin{array}{l}86 \%(12 \text { of } 14 \\
\text { persons) }\end{array}$ & & & $\begin{array}{c}33 \%(1 \text { of } 3 \\
\text { persons })\end{array}$ & & & 0.05 \\
\hline Complications & & & & & & & \\
\hline Pneumonia at RMRSIS admission & $9 \%(n=2)$ & & & $5 \%(n=1)$ & & & 0.67 \\
\hline New diagnosis of pneumonia & $13 \%(n=3)$ & & & $5 \%(n=1)$ & & & 0.39 \\
\hline Chest tubes & 0 & & & $5 \%(n=1)$ & & & 0.27 \\
\hline ARDS & $4 \%(n=1)$ & & & 0 & & & 0.36 \\
\hline
\end{tabular}

respiratory rate), and freedom from such complications as excessive secretions and fever. Finally, the patient had to be emotionally ready to consent to and embrace the weaning program. ${ }^{21}$ The weaning process itself utilized Progressive Ventilator Free Breathing (PVFB), also referred to as 'T-piece' weaning.

With all of the above conditions met, weaning typically began with removal from the ventilator for 2 min of independent, self-initiated breathing. A respiratory care professional was present, and at least one set of parameters were obtained during the wean, including: forced vital capacity, tidal volume, respiratory and heart rate, oxygen saturation, minute volume, negative inspiratory pressure, and end tidal $\mathrm{CO}_{2}$ $\left(\mathrm{ETCO}_{2}\right)$. The regular protocol called for three weans per day, with wean durations (5 mins; 10 mins; 20 mins; 30 mins; $1 \mathrm{~h} ; 2 \mathrm{~h} ; 3 \mathrm{~h}$ ) being increased every 1-3 days, dependent upon the above values and patient comfort and success. Further progression called for 4 and 5 weans twice daily, followed by $12 \mathrm{~h}$ of ventilator free breathing in a $24 \mathrm{~h}$ period, which was then further increased by $2 \mathrm{~h}$ every $1-3$ days until complete freedom from the ventilator was achieved.
While the individuals included in this study were not prospectively randomized into treatment conditions, the two groups were compared on a variety of factors and were found to be equivalent on several important criteria. In addition to their similar neurologic and functional profiles, both groups were admitted with almost identical spontaneous vital capacities, averaging about 0.71 . With respect to several other key variables, they were also similar. There were no significant differences with respect to gender. Five of the 42 participants were women; four of these were in the HTV group $(P=0.096)$. The two groups also did not differ with respect to mean age or duration of injury, as is illustrated in Table 1. To establish the significance of these differences-and to analyze the study's results-independent sample twotailed $t$-tests for comparing means, and Chi squares for comparing frequencies, were used.

\section{Results}

The HTV group achieved significantly faster weaning times (37.6 days vs 58.7 days; $P=0.02$ ). This 21 day difference was observed despite the fact that both 
groups had equivalent tidal volumes at the beginning of their rehabilitation programs, weaned using the same methodology (PVFB), and increased their spontaneous vital capacities to nearly identical levels by the time weaning had been completed (approximately 1.51 on average).

There also were differences in the frequency of atelectasis, with the two groups differing considerably at several points throughout the study. At admission to the RMRSIS, $39 \%$ of the patients who became the LTV group had atelectasis documented on chest X-ray films compared with $84 \%$ of those who ended up in the higher volume group $(P=0.002)$. By the start of each individual's weaning process, approximately half of both groups had atelectasis $(48 \%$ of LTV group, $53 \%$ of HTV group), but by 2 weeks after admission the rate of atelectasis had reversed so that the lower volume group now had more atelectasis (52\%) compared with $16 \%$ of the HTV group $(P=0.01)$ (Table 1).

The higher volumes not only appeared to clear atelectasis more quickly but also to prevent the new onset of atelectasis. While $86 \%$ of the LTV groupmembers who had not had atelectasis upon admission to the RMRSIS developed new atelectasis during their admission, only $33 \%$ of the HTV group developed the new onset of atelectasis $(P=0.05$; Table 1$)$. There were relatively few other respiratory complications as well. In the HTV group there were three: one individual required chest tubes subsequent to pneumothorax which occurred after placement of a subclavian catheter; two individuals had pneumonia-one upon admission to the RMRSIS, one during the hospital stay. In the LTV group six individuals had complications: there was one case of ARDS, and five pneumonias, two which were present upon admission and three of which developed during the RMRSIS hospital stay.

Finally, the two groups differed significantly in their average peak pressure. Peak pressures for the LTV group averaged $28.8 \mathrm{cmH}_{2} \mathrm{O}$ while the higher volume group averaged $35.4 \mathrm{cmH}_{2} \mathrm{O}(P<0.001)$. Additionally, as Table 1 shows, average dynamic compliance for the lower volume group was $41 \mathrm{ml} / \mathrm{cmH}_{2} \mathrm{O}$, compared with $50 \mathrm{ml} / \mathrm{cmH}_{2} \mathrm{O}$ for the HTV group.

\section{Discussion}

Clearly, the time required to wean from the mechanical ventilator was shorter and atelectasis rates were lower in the group for whom high ventilator volumes were used. While the patients who entered the high volume group had more initial atelectasis, their group demonstrated quicker clearing of existing atelectasiswith no evidence of barotrauma-and better prevention of new onset atelectasis. The resultant 3 week difference in weaning time translates into substantial savings in health care costs.

Although it seems very likely that ventilator tidal volume accounted for the difference between the two groups-which were equivalent with respect to weaning methodology and initial tidal volumes and peak flow rates - it is still difficult to determine the true cause of the high volume group's better outcomes. Perhaps larger tidal volumes give paralyzed patients more expanded lung to work with. This could be reflected by the observed increases in lung compliance and the decreased rates of atelectasis. Indeed, as larger volumes recruit additional lung elements and as atelectasis decreases, improved compliance may lead to a greater ease of breathing and to greater success in weaning. On the other hand, perhaps the higher pressures-occurring subsequent to the larger volumes and faster flow rates-were in fact responsible for the rapid clearing of atelectatic lungs rather than the higher volumes themselves. Finally, the gradual approach to increasing volume may have played a key role. Perhaps, by allowing surfactant production to keep pace, re-expanded lung areas are protected from barotrauma and other insults while the simultaneously improving compliance eases the act of breathing and facilitates weaning. Moreover, by using greater lung inflation (through large tidal volumes) and maximizing deflation (by not using PEEP), perhaps the stretching of type II alveolar cells is maximized, stimulating the release of surfactant. In any case, this research does suggest that the physiological processes that often contraindicate the use of high ventilator volumes in non-spinal cord injured people who have diseased lungs or impaired respiratory systems may not be the same in individuals with high-level, otherwise uncomplicated tetraplegia. Certainly, many factors make the individual with tetraplegia different. Tetraplegics differ from other patients, for example, in the paralysis of their intercostal, and in some cases, their diaphragmatic muscles, which makes them highly susceptible to atelectasis. ${ }^{8}$ Atelectasis responds to higher ventilator volumes, probably because of the combination of enhanced release of surfactant which prevents further alveolar collapse, ${ }^{15-17}$ and the recruitment of additional lung elements. ${ }^{18}$ Moreover, tetraplegics spend much of their time supine, a position that reportedly requires larger ventilator volumes to insure ventilation and perfusion of basilar lung regions and further prevent atelectasis and respiratory complications. ${ }^{6}$

The findings of this research are clouded somewhat by the fact that there were not wide differences in the volumes used with the LTV and HTV groups. Not only did the LTV group include individuals whose ventilator volumes were still relatively high, but there were no participants in this study who were treated at the RMRSIS with volumes at or below the $10 \mathrm{ml} / \mathrm{kg}$ recommended by Bezzant and Mortensen. ${ }^{9}$ This was due, in large part, to the previous research at the RMRSIS demonstrating that larger volumes are associated with more rapid clearing of atelectasis. ${ }^{8}$ As a result of this research, larger ventilator volumes have been an established standard of care by all pulmonologists at the RMRSIS. 
Another limitation of this study is that many subjects came to the RMRSIS from other hospitals; thus, consistent information for the coding of both pre-injury and pre-RMRSIS characteristics and complications was not available. Also, because this study was conducted retrospectively, its two study groups were not randomly assigned. However, all subjects did have equivalent neurological levels and equivalent initial and final vital capacities, indicating equivalent weaning potential. Indeed, if any bias existed, it should logically have favored the low volume group with its initially lower incidence of at least one complication that significantly deters successful weaning-atelectasis. Other complications, like pneumonia, ARDS and pneumothorax, were few and differences between the two groups were not significant.

A final limitation of this study relates to the size of its sample. Although the RMRSIS attracts high level tetraplegics from throughout the US and the world, the number of individuals sustaining this catastrophic injury are relatively small. Clearly, further multi-center research involving larger numbers of patients is indicated so respiratory weaning protocols, like the one described here, can be tested and evidence of the role of high volumes in clearing and preventing atelectasis can be further examined.

\section{Acknowledgements}

This research was supported, in part, by funding from the US Department of Education's National Institute on Disability and Rehabilitation Research, Special Projects and Demonstrations for Spinal Cord Injuries, grant \#H133N5001.

\section{References}

1 Goldstone J, Mosham J. Assisted ventilation: weaning from mechanical ventilation. Thorax 1991; 46: 56-62.

2 Wicks AB, Menter RR. Long-term outlook in quadriplegic patients with initial ventilator dependency. Chest 1986; 90: $406-$ 410.
3 Peterson $\mathrm{W}$ et al. Two methods of weaning persons with quadriplegia from mechanical ventilators. Paraplegia 1994; 32: $98-103$.

4 Lamid S, Reaglie GF, Welter K. Respirator-dependent quadriplegics: problems during the weaning period. J Am Paraplegia Soc 1985; 8: $33-37$.

5 Bach JR, Alba AS. Noninvasive options for ventilatory support of the traumatic high level quadriplegic. Chest 1990; 98: 613619.

6 Bynum LJ, Wilson JE, Pierce AK. Comparison of spontaneous and positive-pressure breathing in supine normal subjects (abstract). Proceedings of American Thoracic Society/American Lung Association. Annual Meeting May 16-19, 1976; p. 150.

7 Crane RD, Shapiro BA. Mechanical ventilatory support. JAMA 1985; 254: $235-236$.

8 Peterson P. Pulmonary physiology and medical management. In Whiteneck G, Adler C, Carter RE, et al, eds. The Management of High Quadriplegia. 1989; Demos, New York.

9 Bezzant TA, Mortensen JD. Risks and hazards of mechanical ventilation: a collective review of published literature. Disease- $a$ month 1994; XL: 583-638.

10 Sladen A, Laver MD, Pontoppidan H. Pulmonary complications and water retention in prolonged mechanical ventilation. NEJ Med 1968; 279: $448-453$.

11 Kolbow et al. Severe impairment of lung function induced by high peak airway pressure during mechanical ventilation. Am Rev Respir Dis 1987; 135: $313-315$.

12 Colbridge TC et al. Adverse effects of large tidal volume and low PEEP in canine acid aspiration. Am Rev Respir Dis 1990; 142: $311-315$.

13 Dreyfuss et al. High inflation pressure pulmonary edema. Am Rev Respir Dis 1988; 137: 1159-1164.

14 Dreyfuss D et al. Intermittent positive pressure hyperventilation with high inflation pressure produces pulmonary microvascular injury in rats. Am Rev Respir Dis 1985; 132: 880-884.

15 Massaro GD, Massaro D. Morphologic evidence that large inflations of the lung stimulate secretion of surfactant. Am Rev Resp Dis 1983; 127: 235-236.

16 Faridy EE. Effect of distension on release of surfactant in exercised dogs' lungs. Respir Physiol 1976; 27: 99-114.

17 Nicholas TE, Barr HA. The release of surfactant in rat lung by brief periods of hyperinflation. Respir Physiol 1983; 52: 69-83.

18 Rothen $\mathrm{HU}$ et al. Re-expansion of atelectasis during general anesthesia: a computed tomography study. Br J Anaesth 1993; 71: $788-795$.

19 Daniels L, Worthingham C. Muscle testing: techniques of manual examination. 3rd edn. 1972; Philadelphia: Saunders.

20 Peterson P, Brooks CA, Mellick D, Whiteneck G. Protocol for ventilator management in high tetraplegia. Topics in Spinal Cord Injury Rehabilitation 1997; 2: $101-106$.

21 Johnson K, Grant T, Peterson P. Ventilator weaning for the patient with high-level tetraplegia. Topics in Spinal Cord Injury Rehabilitation 1997; 2: $11-20$. 